\title{
A Secure Delegation Process using Diffie-Hellman Assumption in Cloud Computing
}

\author{
Sarath Kumar Reddy G. \\ Final Year, B. Tech \\ School of Computing Science and \\ Engineering \\ VIT University, Vellore-14, India
}

\author{
Anish Varsha I. \\ Final Year, B. Tech \\ School of Computing Science and \\ Engineering \\ VIT University, Vellore-14, India
}

\author{
Sai Praneeth R.V.C \\ Final Year, B. Tech \\ School of Computing Science and \\ Engineering \\ VIT University, Vellore-14, India
}

\begin{abstract}
Data owner encrypt their data before outsourcing into the cloud for the purpose of privacy preserving. Additionally, the attribute based encryption method is used to enhance the confidentiality and access control. Delegation is a process which is performed by the users who are containing the less computing power. They delegate their decryption process to the cloud server to reduce the computation cost. This time the cloud server may change the given cipher text and sends the modified one to the data user for malicious attack. This time the access control cannot be malleable. To enhance the access control, we propose a circuit cipher text-policy attribute-based hybrid encryption with verifiable delegation method. For the authentication purpose, mac mechanism is added with the symmetric encryption technique. By this mechanism, we can certify the confidentiality of data, accuracy of the delegated computing results and enhance the access control. Our paper uses the k-multi linear Decisional Diffie-Hellman algorithm to improve the security to the encrypted data. This scheme takes only less computational and communication cost so it will be done at practically.
\end{abstract}

\section{Keywords}

Attribute based encryption, data sharing, verifiable delegation, authentication, confidentiality.

\section{INTRODUCTION}

The appearance of cloud computing transports a radical novelty to the organization of the data possessions within this calculating surroundings, the cloud servers can present different data services, such as isolated data storage and outsourced allocation calculation etc. For information cargo space, the servers amass a huge quantity of communal information, which might be accessed by certified users. For allocation calculation, the servers could be accustomed to hold and determine frequent data dealing to the user's burden. As appliances shift to cloud computing proposals, verifying delegation process using cipher text-policy attribute-based encryption (CP-ABE) is used to guarantee the data privacy and the verifiability of allocation on untruthful cloud servers. Captivating health check data distribution as an example. among the rising volumes of health check images and health check records, the medical care associations set a big amount of data in the cloud for dropping data cargo space expenses and maintaining health assistance.

Since the cloud server may not be believable, the file cryptographic cargo space is an efficient technique to intercept secret data from human being stolen or interfered. In the intervening time, they might require to split data with the human being who satisfies a number of necessities. The necessities, i.e., right to use strategy, might be $\{$ health check organization relationship $\wedge$ (presence doctor of medicine $\vee$ leader doctor of medicine) $\wedge$ Orthopedics $\}$. To compose such data distribution is attainable, attribute-based encryption is appropriate. Here are two corresponding forms of attribute based encryption. Lone is key-policy attribute-based encryption (KP-ABE) and the additional is cipher text-policy attribute-based encryption (CPABE). In a KP-ABE scheme, the conclusion of right of entry strategy is completed by the key dispenser as a substitute of the enciphered, which restrictions the possibility and usability for the scheme in sensible submissions.

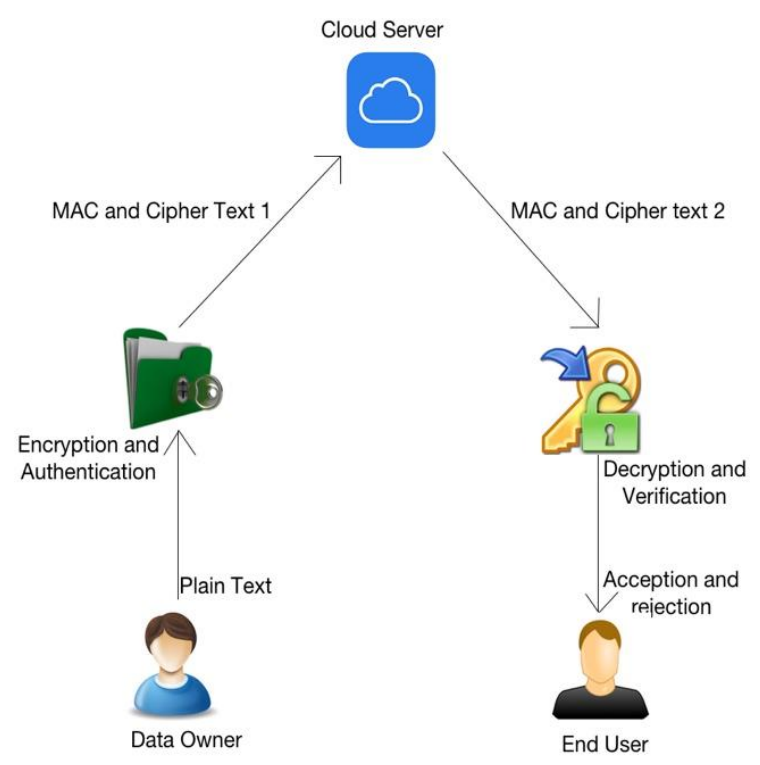

Figure1.data sharing system

On top of the divergent, in a CP-ABE scheme, each cipher text is linked with a right of entry organization, and every personal key is tagged by way of a position to expressive aspects. A user is clever to make intelligible a cipher text if the key's aspect position satisfies the right of entry arrangement connected with a cipher text. It seems that, this scheme is theoretically quicker to conventional right of entry manage techniques. Alternatively, inside an ABE system, the right of entry strategy for universal circuits possibly will be observed as the physically powerful form of the strategy appearance that circuits can converse any line up of fixed in succession time. Allocation calculating is one more major service provided by the cloud servers. During over the circumstances, the medical care associations pile up data records in the cloud with using $\mathrm{CP}-\mathrm{ABE}$ below convinced right of entry strategies. The users, 
who desire to right to use the data records, decide not to switch the compound procedure of decryption nearby owing to imperfect possessions. As a substitute, they are the majority possible to outsource fraction of the decryption procedure to the cloud server. While the mistrustful cloud servers who can interpret the innovative cipher text into an uncomplicated one. Could be accomplished not anything about the plaintext on or after the allocation. The labor of allocation is talented however predictably suffers from two troubles.

a. The cloud server strength interferes or substitutes the data owner's innovative cipher text for spiteful attacks, and then responds a fake distorted cipher text.

b. The cloud server force tricks the endorsed user for cost reduction. Despite the fact that the servers could not react a correct distorted cipher text to a dishonest user, he could trick an official one that he/she is not qualified.

Additional, throughout the deployments of the cargo space and allocation armed forces, the main necessities of this investigate are obtainable as follows. 1) Privacy (in distinguishability below discriminating chosen plaintext attacks (IND-CPA)). With the cargo space examine offered by the cloud server, the outsourced data be supposed to not be escaped even if malware or hackers penetrate the server. Besides, the unconstitutional users with no sufficient elements to satisfy the right to use strategy could not entree the plaintext of the statistics. Furthermore, the unconstitutional admission from the hopeless server who gets an additional alteration key ought to be prohibited. 2) Verifiability. Throughout the allocation calculation, a user possibly will authenticate whether the cloud server reacts a correct distorted cipher text to assist him/her decrypt the cipher text straight away and properly. Namely, the cloud server could not act in response a false distorted cipher text or trick the official user that he/she is illegal. Thus, in this paper, we will effort to process the description of CP-ABE with supportable allocation in the cloud to believe the data privacy, the fine-grained data entrée manages and the verifiability of the allocation. The associated refuge description and INDCPA refuge game used in the evidence are obtainable in to describe the over attacks of the adversaries.

\section{RELATED WORK}

We concentrated on strategies diagonally manifold establishment and the problem of what terminologies could accomplish. In recent period, raised a structure for understanding KPABE for universal circuits. Previous to this technique, the well-built form of appearance is Boolean formulas in $\mathrm{ABE}$ systems, which is at rest a distant weep from being clever to articulate right of entry, manage in the form of any agenda or route. Essentially, in attendance at a standstill stay behind two harms. The primary one is their have no creation for realizing CPABE for universal circuits, which is theoretically closer to conventional entrée manage. The further is associated to the effectiveness, since the outlet circuit $\mathrm{ABE}$ scheme is immediately a small piece encryption one. Thus, it is actually still leftovers an essential unlock trouble to design a professional circuit $\mathrm{CP}-\mathrm{ABE}$ scheme. Further proposed the basic KEM/DEM structure for hybrid encryption which preserve encrypt messages of random distance end to end. Based on their clever work, a one-time MAC was collective with symmetric encryption to expand the KEM/DEM representation for hybrid encryption.

Such enhanced representation has the benefit of achieving superior refuge necessities ABE with Verifiable allocation. Since the opening of ABE, there have been advances in manifold instructions. The submission of outsourcing calculation is one of a significant way. Then intended the first ABE through outsourced decryption scheme to decrease the calculation cost for the duration of decryption. After that, proposed the description of $\mathrm{ABE}$ with demonstrable outsourced decryption. They request to assurance the precision of the unique cipher text by using a promise. However, while the data owner creates an assurance without any top secret value regarding his individuality, the hopeless server can then fake a assurance for a message he decides. Thus the cipher text connecting to the message is at danger of being interfered. In addition, just transform the assurances for the cipher text connecting to the message is not sufficient. The cloud server can mislead the user with appropriate agreements by react the terminator to trick that he/she is not permitted to right of entry to the data.

\section{OUR CONTRIBUTION}

Encouraged by the necessities in the cloud, we change the representation of $\mathrm{CP}-\mathrm{ABE}$ with verifiable allocation and there a physical structure to understand circuit's cipher text-policy standard hybrid encryption with verifiable delegation (VDCPABE). To maintain data confidential and attain fine grain right of entry control, our preliminary point is a circuit keypolicy attribute-based encryption. We provide the anticollusion circuit $\mathrm{CP}-\mathrm{ABE}$ structure in this document for the basis that $\mathrm{CPABE}$ is theoretically earlier to the conventional right of entry control methods. For the main competence problems of $\mathrm{ABE}$, preceding structures provided an alert technique to outsource the majority transparency of decryption to the cloud. However, there is no assurance that the intended result revisited by the cloud is always accurate. The cloud server may fake cipher text or cheat the qualified user that he still does not have agreements to decryption. To authenticate the correctness, we make bigger the $\mathrm{CP}-\mathrm{ABE}$ cipher text into the attribute based cipher text for two corresponding policies and put in a MAC for every cipher text, so that whether the user has agreements he/she could gain a confidentially established key to verify the exactness of the allocation and prevent from imitation of the cipher text. Aspiring at additional improving the competence and providing spontaneous explanation of the refuge proof, the notion of hybrid encryption is too introduced in this work. Besides, refuge of the VDCPABE system guarantees that the hopeless cloud will not be clever to study whatever thing about the encrypted message and fake the unique cipher text. After that, the proposed scheme is replicated in the GMP library. Finally; the system is accomplished to be sensible in the cloud.

\subsection{Our Techniques}

Verifiable delegation (VD) is used to defend approved users from human being tricked throughout the delegation. The data holder encrypts his memorandum beneath right of entry strategy then computes the accompaniment circuit, which productions the reverse bit of the output, and encrypts an arbitrary constituent of the equal length under the policy. The users can then contract out their multipart right of entry control strategy choice and fraction process of decryption to the cloud. Such extensive encryption guarantees that the users can get also the memorandum or the arbitrary constituent, which evades the circumstances when the cloud server misleads the users that they are not contented to the right of entry strategy, however, they get together the right of entry strategy in point of fact. In CP-ABE we use a hybrid variation for two causes: one is to the circuit $\mathrm{ABE}$ is a small piece encryption, and the additional is that the verification of the delegated cipher text should be assured. The cipher text of the hybrid VD-CPABE system is separated into two mechanisms: the CP-ABE for 
circuits and constructs the key encapsulation mechanism (KEM) part, and a symmetric encryption benefit the encryptthen-Mac mechanism construct the genuine encryption mechanism (AE) part. Each KEM encrypts a accidental group constituent and subsequently plots it by way of key origin functions into a symmetric encryption key and a one-time established key. Then the arbitrary encryption key is used to encrypt the memorandum of any distance end to end. Established key and the data owner's ID are used to authenticate the MAC of the cipher text. Only when the server dose not fake the unique cipher text and react an accurate fractional decrypted cipher text, the user could be clever to correctly authenticate the MAC. On behalf of realization, the current work on multi linear maps above the integers is useful to replicate the system in the GMP library in VC 6.0. While the operation time for the coupling in the multi linear plot is much more than the one in the bilinear plot, we could accomplish the strongest universal circuit's right of entry strategy up to at the moment. As well, through using verifiable delegation, the process time for the user is small and self-governing of the difficulty of the circuit. For the refuge, we show that the INDCPA secure KEM joins with the IND-CCA secure validated (symmetric) encryption system acquiesces our IND-CPA secure hybrid VD-CPABE system.

\subsection{Organization}

During the following section, we explain various associated mathematical troubles. A proper meaning of hybrid VD$\mathrm{CPABE}$ and its equivalent refuge model is given in below sections. We propose an actual structure for VD-CPABE and we examine the security of the proposed method. Consequently, we present a concise presentation analysis.

\section{NOTATION}

Here the break of the article we permit $\mathrm{Zp}$ be a fixed field with primary order $\mathrm{p} . \perp$ is an official symbol indicates termination. If $X$ is a fixed set, then $x \leftarrow X$ indicates that $x$ is arbitrarily chosen from $\mathrm{X}$. If $\mathrm{A}$ is an algorithm, then $\mathrm{A}(\mathrm{x}) \rightarrow \mathrm{y}$ indicates that $\mathrm{y}$ is the production by organizing the algorithm on contribution $x$. We indicate $G(\lambda, k)$ as a group making algorithm where $\lambda$ is the refuge parameter and $\mathrm{k}$ is the amount of permissible coupling operation. As normal, a utility $\varepsilon: \mathrm{Zp} \rightarrow$ $\mathrm{R}$ is insignificant if for every $\mathrm{c}>0$ there is a $\mathrm{K}$ such that $\varepsilon(\mathrm{k})<$ $\mathrm{k}^{-\mathrm{c}}$ for all $\mathrm{k}>\mathrm{K}$.

\subsection{System Description and Assumption}

As exposed in Table 1, the functions in the VD-CPABE structure are initially summarized. In the scheme, the data holder and the consumers are equally registered beings and got private keys from the influence. The influence is invented to be the only gathering that is completely hopeful by all contributors.

Table 1 Function Description

\begin{tabular}{ll}
\hline Role & Description \\
\hline Authorithy & $\begin{array}{c}\text { Attribute Key generator center } \\
\text { (trusted third party) }\end{array}$ \\
End Uner & $\begin{array}{c}\text { Encrypting party who uploads his } \\
\text { encrypted data to the cloud }\end{array}$ \\
Cloud Server & $\begin{array}{c}\text { Decrypting party who outsources } \\
\text { the most overhead computation } \\
\text { in the cloud }\end{array}$ \\
The party who provides storage and \\
outsourced computation services
\end{tabular}

Like to the preceding schemes the server is invented to be hopeless. Resonance faith management principles as well as inspecting principles could be worn to establish high-quality business associations between the cloud server and the user. According to this structure, the cloud server could be considered as an honest cloud service contributor. In fact, the role-based right of entry management is proposed based on this statement. Yet, by means of this single method, we will be at the dangers of unidentified attacks and the obtainable of the spiteful system supervisor, which may consequence in data outflow, termination of right of entry manage and breakdown of outsourcing. As well, faith management method may source an additional workload for the examiner. Thus, it is highminded time to make a sensible cryptography scheme to defend data and manage entrée with a hopeless server.

\subsection{Circuits}

In the perspective, we still confine our concentration to the monotone Boolean circuit with a solitary production gate. The explanation of a circuit and its estimation are as follows.

Definition 1. A solitary production circuit is a 5-tuple $\mathrm{f}=(\mathrm{n}, \mathrm{q}$, $\mathrm{A}, \mathrm{B}, \mathrm{G})$. Here $\mathrm{n}$ is the quantity of inputs, $\mathrm{q}$ is the quantity of gates, and $\mathrm{n}+\mathrm{q}$ is the quantity of wires. Let Inputs $=\{1,2,3 \ldots$, $n\}$, Wires $=\{1,2,3 \ldots n+q\}$, Gates $=\{n+1, n+2, \ldots, n+q\}$ and Output Wire $=\{\mathrm{n}+\mathrm{q}\}$. Then A: Gates $\rightarrow$ Wires/Output Wires is a task to find each gate's primary inward bound wire, B: Gates $\rightarrow$ Wires/Output Wires is a task to find each gate's secondary inward bound wire and G: Gates $\rightarrow\{$ AND, OR $\}$ is a task to find a gate as also an AND or OR gate. Gates have two inputs, random functionality and a solitary broaden every noninput wire is the sociable wire of various gates. We need A (w) $<\mathrm{B}(\mathrm{w})<\mathrm{w}$ for all $\mathrm{w} \in$ Gates. Let intensity (w) colleagues to the extent of the nearest pathway to an input wire plus 1 and if $\mathrm{w} \in$ Inputs subsequently strength $(\mathrm{w})=1$. We explain the assessment of the circuit $\mathrm{f}$ as $\mathrm{f}(\mathrm{x})$ on input the cord $\mathrm{x} \in\{0,1\}$ $n$, and permit $f_{w}(x)$ be the price of wire $w$ on input $x$. Given the monotone Boolean circuit $f$ we can calculate its accompaniment circuit $\mathrm{f}$, which outputs the reverse bit of the output of $f$. For the circuit $f$, cancellation gates will stay behind only at the input intensity by applying De Morgan's rule. We will disregard the deepness of the cancellation gates. See Fig.2 for a figure of a circuit $f$ and the equivalent accompaniment circuit $\mathrm{f}$.

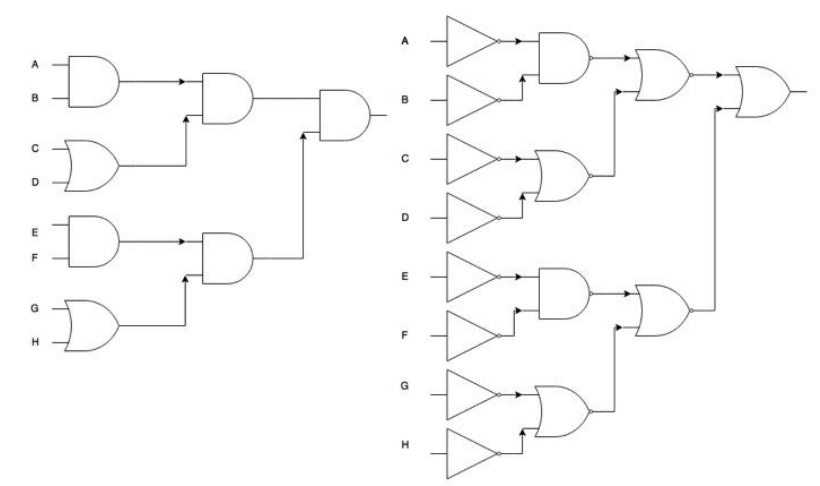

Figure.2 Left: $A$ predictable circuit $\mathbf{f}=(\mathrm{A} \vee \mathrm{B}) \wedge(\mathbf{C} \wedge \mathrm{D})$ $\wedge(\mathbf{E} \vee \mathbf{F}) \wedge(\mathbf{G} \vee \mathbf{H})$. Right: An accompaniment circuit equivalent to the left circuit $\mathbf{f}=(\mathbf{A} \wedge \mathbf{B}) \vee(\mathbf{C} \vee D) \vee(\mathbf{E} \wedge$ F) $\vee(\mathbf{G} \wedge \mathbf{H})$

\subsection{Multilinear Map}

Definition 2. It sprints $\mathrm{G}(\lambda, \mathrm{k})$ and outputs $\mathrm{k}$ repeated groups of the same main order $p$. Let the fundamentals be the producers of the above groups and set $g=g 1$. After that be 
present a set of bilinear maps (write as e for easy) that has the following possessions. For $\mathrm{a}, \mathrm{b} \leftarrow \mathrm{Zp}$, we have $\mathrm{e}\left(\mathrm{g}^{\mathrm{a}}{ }_{\mathrm{i}}, \mathrm{g}^{\mathrm{b}} \mathrm{j}\right)=$ $\mathrm{g}^{\mathrm{ab}} \mathrm{i+j}$.

Definition 3. (k- Multi linear Decision-Diffie-Hellman problem). A competitor runs $\mathrm{G}(\lambda, \mathrm{k})$ to get a series of groups of major order $\mathrm{p}$ where every comes with a canonical initiator $\mathrm{g}$ $=\mathrm{g} 1, \mathrm{~g} 2 \ldots, \mathrm{gk}$. Then it selects $\mathrm{s}, \mathrm{c}, \mathrm{c} 1, \ldots, \mathrm{ck} \leftarrow \mathrm{Zp}$. The benefit in characteristic the tuple $\left(g, g^{s}, g^{c 1}, \ldots, g^{c k}, g_{k}{ }^{s} \Pi j \in[1 ; k] c_{j}\right)$ from $\left(\mathrm{g}, \mathrm{g}^{\mathrm{s}}, \mathrm{g}^{\mathrm{cl}}, \ldots, \mathrm{g}^{\mathrm{ck}}, \mathrm{g}_{\mathrm{k}}^{\mathrm{c}}\right)$ is unimportant in $\lambda$.

\section{OUR MODEL OF HYBRID VD-CPABE}

In this segment, we present the description and protection model of our fusion VD-CPABE. In such a method, a circuit ciphertext-policy attribute-based encryption system, a symmetric encryption system and an encrypt-then-mac method are useful to make sure the privacy, the fine-grained right of entry manage and the verifiable delegation.

\subsection{Hybrid vd-cpabe}

Definition 4. A hybrid VD-CPABE system is distinct through a tuple of algorithms (arrangement, fusion Encrypt, Key- Gen, Transform, and Verify-Decrypt). The explanation of all algorithms is as follows.

- $\quad \operatorname{Setup}(\lambda, \mathrm{n}, 1)$. Performed by the influence, this algorithm takes as input a refuge parameter $\lambda$, the quantity of attributes $n$ and the greatest depth 1 of a circuit. It yields the public constraints PK and a master input MK which is reserved top secret.

- Fusion Encrypt (PK, M, f). This algorithm is performed by the data owner. It could be expediently separated into two fractions: Key Encapsulation Mechanism (KEM) and valid symmetric encryption (AE). The KEM algorithm gets as input the public parameters PK and a right of entry structure $f$ for circuit. It calculates the difficult circuit $f$ and selects an arbitrary string $\mathrm{R}$. Then it constructs $\mathrm{K}_{\mathrm{M}}=$ $\left\{\mathrm{dk}_{\mathrm{m}}, \mathrm{vk}_{\mathrm{m}}\right\}, \mathrm{K}_{\mathrm{R}}=\left\{\mathrm{dk}_{\mathrm{r}}, \mathrm{vk}_{\mathrm{r}}\right\}$ and the CP-ABE ciphertext $\left(\mathrm{CK}_{\mathrm{M}}, \mathrm{CKR}\right)$. The $\mathrm{AE}$ algorithm obtains as input a message $M$, the arbitrary string $R$, the symmetric key $K_{M}$ and $\mathrm{K}_{\mathrm{R}}$. Then it outputs the ciphertext $\left(\mathrm{C}_{\mathrm{M}}, \mathrm{C}_{\mathrm{r}}, \sigma_{\mathrm{ID}}\right.$; $\left.v k_{m}\left(C_{M} \| C_{R}\right), \sigma_{I D} ; v_{r}\left(C_{M} \| C_{R}\right)\right)$. The entire ciphertext for our VD-CPABE system is the tuple $\mathrm{CT}=\left(\mathrm{CK}_{\mathrm{M}}, \mathrm{CK}_{\mathrm{R}}, \mathrm{C}_{\mathrm{M}}\right.$, $\left.\mathrm{C}_{\mathrm{R}}, \sigma_{\mathrm{ID}} ; \mathrm{vk}_{\mathrm{m}}\left(\mathrm{C}_{\mathrm{M}} \| \mathrm{C}_{\mathrm{R}}\right), \sigma_{\mathrm{ID}} ; \mathrm{vk}_{\mathrm{r}}\left(\mathrm{C}_{\mathrm{M}} \| \mathrm{C}_{\mathrm{R}}\right)\right)$

- KeyGen (MK, $\mathrm{x} \in\{0,1\} \mathrm{n})$. The influence produces private keys for the users. This algorithm obtains as input the master key MK and a bit string x. It outputs a private key SK and a conversion key TK.

- Transform (TK, CT). Implemented by the cloud servers, this algorithm obtains as input the alteration key TK and a cipher text $\mathrm{CT}$ that was encrypted underneath $\mathrm{f}$ and $\mathrm{f}$. It outputs the incompletely decrypted cipher text $\mathrm{CT}^{\prime}=\left(\mathrm{CK}^{\prime}\right.$ $\left.{ }_{M}, C_{m}, C_{R}, \sigma_{I D} ; v_{m}\left(C_{M} \| C_{R}\right)\right)$ or $C^{\prime}=\left(C^{\prime}{ }_{R}, C_{M}, C_{R}\right.$, $\left.\sigma_{\mathrm{ID}} ; \mathrm{vk}_{\mathrm{r}}\left(\mathrm{C}_{\mathrm{M}} \| \mathrm{C}_{\mathrm{R}}\right)\right)$.

- Verify-Decrypt (SK, $\left.\mathrm{CT}^{\prime}\right)$. Performed by the users, this algorithm obtains as inputs the secret key SK and the incompletely decrypted cipher text $\mathrm{CT}^{\prime}$. Firstly, it authenticates the authority of $\sigma$. Then it outputs the message $\mathrm{Mb}$, which convince that if $\mathrm{f}(\mathrm{x})=1$ then $\mathrm{Mb}=\mathrm{M}$ and if $\mathrm{f}(\mathrm{x})=0$ then $\mathrm{Mb}=\mathrm{R}$.

\subsection{Security model}

While we utilize Key Encapsulation Mechanism (KEM) and Authenticated Encryption (AE) to construct our hybrid VDCPABE system, we explain the security description independently at first. The privacy property (in distinguishability of encryptions under discriminating selected plaintext attacks (IND-CPA)) necessary for Key Encapsulation Mechanism (KEM) is captured by the subsequent playoffs nearby challenger $\mathrm{A}$.

\section{Game.KEM}

- Init. The challenger gives a dispute entrée structure $f *$, where it needs to be challenged.

- $\quad$ Setup. The simulator sprints the Setup algorithm and gives the public constraints PK to the challenger.

- KeyGen Queries I. The challenger makes frequent private key queries equivalent to the places of elements $\mathrm{x}_{1}, \ldots, \mathrm{x}_{\mathrm{q} 1}$. We require so as to $\forall \mathrm{i} \in \mathrm{q}_{1}$ we contain $\mathrm{f}^{*}\left(\mathrm{x}_{\mathrm{i}}\right)=0$.

- $\quad$ Encrypt. The simulator encrypts $\mathrm{K}_{0}$ below the structure $\mathrm{f}^{*}$, arbitrary decides $\mathrm{K}_{1}$ from key space and flips an arbitrary coin $b$. Then the simulator flings $K_{b}$ and the ciphertext $\mathrm{CK}^{*}$ to the challenger.

- KeyGen Queries II. The challenger constructs frequent private key queries equivalent to the sets of attributes $\mathrm{x}_{\mathrm{q} 1}$, $\ldots, \mathrm{x}_{\mathrm{q}}$ where $\mathrm{f}^{*}(\mathrm{x})=0$.

- Guess. The challenger outputs an estimate $b^{\prime}$ of $b$. We describe the benefit of a challenger $A$ in this game is $\operatorname{Pr}\left[b^{\prime}\right.$ $=\mathrm{b}]-1 / 2$. Then a KEM structure is protected besides discriminating preferred plaintext attacks if the benefit is insignificant. The privacy possessions (in distinguishability of encryptions below discriminating selected ciphertext attacks (IND-CCA)) necessary for AE is captured by the following games beside challenger $\mathrm{A}$.

Game.AE

- Init. The challenger presents two equivalent distance end to end messages $M_{0}$ and $M_{1}$.

- Setup. The simulator sprints the system algorithm and produces the symmetric key KAE.

- Encrypt. The simulator spins an arbitrary coin b, encrypts $\mathrm{Mb}$ beneath the symmetric key KAE, produces the ciphertext $\mathrm{C} *$ and gives it to the challenger.

- Decrypt Queries. The challenger creates frequent decryption queries. When the specified ciphertext $\mathrm{C} \vDash \mathrm{C}^{*}$, the simulator will reverse $\mathrm{D}_{\mathrm{KAE}}(\mathrm{C})$ and $\sigma_{\mathrm{KAE}}(\mathrm{C})$ to the challenger.

- Guess. The challenger outputs an estimate $b^{\prime}$ of $b$. Let $\operatorname{Pr}\left[b^{\prime}=b\right]-1 / 2$ be the benefit of a challenger $A$ in this game. By means of the encrypt-then-mac technique, we declare that an AE method is IND-CCA protected if the benefit is insignificant.

From the above, we here the refuge form for our method as follows.

\section{Game.VD-CPABE}

- Init. The VD-CPABE algorithm challenger presents the dispute right of entry structure $\mathrm{f} *$ and two equivalent distance end to end messages $\mathrm{M}_{0}$ and $\mathrm{M}_{1}$.

- Setup. The simulator sprints the system algorithm and provides the public constraints PK to the challenger.

- KeyGen Queries I. The challenger constructs frequent private key queries equivalent to the locates of elements $\mathrm{x}_{1}, \ldots, \mathrm{x}_{\mathrm{q} 1}$. We necessitate that $\forall_{\mathrm{i}} \in{ }_{\mathrm{q} 1}$ we have $\mathrm{f}^{*}\left(\mathrm{x}_{\mathrm{i}}\right)=0$ 
- Encrypt. The simulator encrypts K0 beneath the organization $\mathrm{f}^{*}$ by means of the KEM algorithm. Then the simulator spins an arbitrary coin $\mathrm{v}$ and encrypts $\mathrm{Mv}$ beneath the symmetric key $\mathrm{K}_{0}$ by means of the $\mathrm{AE}$ algorithm. Then the entire ciphertext is agreed to the VDCPABE algorithm challenger.

- KeyGen Queries II. The challenger creates frequent private key queries equivalent to the locates of qualities $\mathrm{x}_{\mathrm{q} 1}, \ldots, \mathrm{x}_{\mathrm{q}}$ where $\mathrm{f}^{*}(\mathrm{x})=0$.

- Guess. The challenger outputs an estimate $\mathrm{v}^{\prime}$ of $\mathrm{v}$. We describe the benefit of a challenger $\mathrm{A}$ in this game is $\operatorname{Pr}\left[\mathrm{v}^{\prime}\right.$ $=\mathrm{v}]-1 / 2$. We'll illustrate that if a KEM system is INDCPA protected and an AE system is IND-CCA protected then our hybrid encryption system is IND-CPA protected.

\section{OUR HYBRID VD-CPABE SCHEME}

In this section, we propose a concrete circuit ciphertext-policy attribute-based fusion encryption with verifiable allocation scheme based on the multi linear maps and the verifiable computing technology under cloud environment. Authority generates private keys for the data owner and user. The data owner encrypts his data using hybrid encryption system, generates a privately verifiable MAC for each symmetric ciphertext and then uploads the whole ciphertext to the cloud server. Then the data owner could be offline. The user, who wants to access to the data, interacts with the cloud server. In the figure, the dashed arrows indicate that the value is transferred secretly, while the solid arrows indicate that the value is transferred without a secure channel. Using general circuits to express the access control policy, we construct a monotone circuit with depth 1 and input size to be $n$. The proposed hybrid VDCPABE scheme consists of the following probabilistic polynomial time (PPT) algorithms.

- $\operatorname{Setup}(\lambda, \mathrm{n}, \mathrm{l})$. This algorithm is executed by the authority. It obtains as input a refuge constraint $\lambda$, the number $n$ of input dimension and the greatest depth 1 of a circuit. Then it runs $\mathrm{G}(\lambda, \mathrm{k}=l+1)$, outputs a sequence of groups of prime order $\mathrm{p}$ and their corresponding generators $\mathrm{g} 1, \ldots$, $\mathrm{gk}$ and sets $\mathrm{g}=\mathrm{g} 1$. After that it chooses three one-way hash functions $\mathrm{H}_{1}: \mathrm{G}_{\mathrm{k}} \rightarrow\{0,1\}^{\mathrm{m}}, \mathrm{H}_{2}: \mathrm{G}_{\mathrm{k}} \rightarrow \mathrm{Z}_{\mathrm{p}}, \mathrm{H}_{3}:\{0,1\}$ $* \rightarrow \mathrm{G}_{1}$, random $\alpha \in \mathrm{Z}_{\mathrm{p}}, \mathrm{a} \in \mathrm{Z}_{\mathrm{p}}, \mathrm{h}_{11}, \ldots, \mathrm{h}_{1 \mathrm{n}}, \mathrm{h}_{21}, \ldots, \mathrm{h}_{2 \mathrm{n}} \in$ $\mathrm{G}_{1}$ and sets $\mathrm{y}=\mathrm{g}^{\mathrm{a}}$. The public key PK as well as the system master key MK are as follows: $\mathrm{PK}=\left(\mathrm{g}^{\alpha}{ }_{\mathrm{k}}, \mathrm{H}_{1}, \mathrm{H}_{2}\right.$, $\left.\mathrm{H}_{3}, \mathrm{y}, \mathrm{h}_{1}, \ldots, \mathrm{h}_{\mathrm{n}}, \mathrm{h}_{\mathrm{n}+1}, \ldots, \mathrm{h}_{2 \mathrm{n}}\right), \mathrm{MK}=\mathrm{g}^{\alpha}$.

- $\quad$ Fusion Encrypt (PK, $\mathrm{f}=(\mathrm{n}, \mathrm{q}, \mathrm{A}, \mathrm{B}$, Gate Type), $\mathrm{M} \in\{0$, $\left.1\}^{\mathrm{m}}\right)$ ). This algorithm is implemented by the data owner. Taking the public parameters PK, a description $f$ of a circuit and a message $\mathrm{M} \in\{0,1\}^{\mathrm{m}}$ as input, the hybrid encryption algorithm works as follows.

1. It chooses random $\mathrm{R} \in\{0,1\} \mathrm{m}, \mathrm{s} 1, \mathrm{~s} 2, \mathrm{~s} 3 \in \mathrm{Zp}$ and computes $C_{M}^{\prime}=g^{s 1}{ }_{k-1}, r_{1}=H_{2}\left(g^{\text {asl }}{ }_{k}\right), C_{M}=M \oplus H_{1}\left(g^{\alpha s 1}{ }_{k}\right)$, $\mathrm{C}^{\prime}{ }_{\mathrm{R}}=\mathrm{g}_{\mathrm{k}-1}^{\mathrm{s} 2}, \mathrm{r}_{2}=\mathrm{H}_{2}\left(\mathrm{~g}_{\mathrm{ks}}\right), \mathrm{C}_{\mathrm{R}}=\mathrm{R} \oplus \mathrm{H}_{1}\left(\mathrm{~g}_{\mathrm{ks}}{ }_{\mathrm{k}}\right), \sigma_{1}=$ MAC. $\mathrm{Sign}_{\mathrm{IDo}} ; \quad \mathrm{r}_{1} \quad\left(\mathrm{C}_{\mathrm{M}} \| \mathrm{C}_{\mathrm{R}}\right), \quad \sigma_{2}=$ MAC.Sign ${ }_{\text {IDo }} ; r_{2}\left(C_{M} \| C_{R}\right)$. Where $\sigma_{1}=g^{\alpha s 3} y^{\text {ts } 3} \mathrm{H}_{3}{ }^{t{ }^{t 3}}\left(I_{0}\right) H_{3}{ }^{r 1 s 3}($ $\left.\mathrm{ID}_{0}\left\|\mathrm{C}_{\mathrm{M}}\right\| \mathrm{C}_{\mathrm{R}}\right)$ and $\sigma 2=\mathrm{g}^{\alpha \mathrm{ss} 3} \mathrm{y}^{\mathrm{ts} 3} \mathrm{H}_{3}{ }^{\mathrm{ts} 3}\left(\mathrm{ID}_{0}\right) \mathrm{H}_{3}{ }^{\mathrm{r} 2 \mathrm{~s} 3}\left(\mathrm{ID}_{0}\left\|\mathrm{C}_{\mathrm{M}}\right\| \mathrm{C}_{\mathrm{R}}\right)$. Set $\sigma_{\mathrm{M}}=\left\{\sigma_{1}, \mathrm{~g}_{\mathrm{k}}{ }^{\alpha \mathrm{s} 3}, \mathrm{gk}_{-1}{ }^{\mathrm{ts} 3}, \mathrm{H}_{3}{ }^{\mathrm{s} 3},{ }_{\mathrm{k}-1}\left(\mathrm{ID}_{\mathrm{o}}\left\|\mathrm{C}_{\mathrm{M}}\right\| \mathrm{C}_{\mathrm{R}}\right)\right\}$ and $\sigma_{\mathrm{R}}=$ $\left\{\sigma_{2}, \mathrm{~g}_{\mathrm{k}}{ }^{\alpha 3} \backslash, \mathrm{g}_{\mathrm{ks} 3}^{\mathrm{ts}-1}, \mathrm{Hs}^{3}{ }_{3, \mathrm{k}-1}\left(\mathrm{ID}_{\mathrm{o}}\left\|\mathrm{C}_{\mathrm{M}}\right\| \mathrm{C}_{\mathrm{R}}\right)\right\}$. The fractional ciphertext is $\left(\mathrm{C}_{\mathrm{M}}, \mathrm{C}^{\prime}{ }_{\mathrm{M}}, \sigma_{\mathrm{M}}, \mathrm{CR}, \mathrm{C}^{\prime}{ }_{\mathrm{R}}, \sigma_{\mathrm{R}}\right)$. Note to facilitate the significance $\mathrm{g}^{\alpha} \mathrm{y}^{\mathrm{t}}, \mathrm{g}^{\mathrm{t}}$ and $\mathrm{H}_{3}^{\mathrm{t}}\left(\mathrm{ID}_{\mathrm{o}}\right)$ are the private keys for the encrypted shown in the KeyGen algorithm.

2. Agreed the circuit entrée structure $f$, it produces a set off circuit $f$ using De Morgan's rule such that cancellation gates emerge only on the input wires. Obtains $f$ for example, the encryption algorithm chooses arbitrary $r_{1}, \ldots$, $r_{n+q-1} \in Z p$ and lets $r_{n+q}=s 1$. The arbitrariness $r_{w}$ is linked with wire w. We then illustrate how the circuit $f$ splits the encryption proponent s1. The structure of the splits depends on if $w$ is an Input wire, an OR gate, or an AND gate. The circuit metaphors are as follows. - Input wire. For $\mathrm{w} \in[1, \mathrm{n}]$, this algorithm desires arbitrary $\mathrm{z}_{\mathrm{w}}$ $\in \mathrm{Z}_{\mathrm{p}}$. The splits are: $\mathrm{C}_{\mathrm{w}}, 1=\mathrm{y}^{\mathrm{rw}}\left(\mathrm{yh}_{\mathrm{w}}{ }^{-\mathrm{zw}}, \mathrm{C}_{\mathrm{w}, 2}=\mathrm{g}^{\mathrm{zw}}\right.$. Gate OR. Let $\mathrm{j}=$ depth (w). This algorithm decides arbitrary aw $\in \mathrm{Zp}$. The splits are: $\mathrm{C}_{\mathrm{w}, 1}=\mathrm{g}_{\mathrm{w}}^{\mathrm{a}}, \mathrm{C}_{\mathrm{w}} 2=\mathrm{g}$ $\underset{w}{a(r-a r A(w))} j, C_{w}, 3=g{ }^{a}\left(r_{w}-a_{w} r_{B}(w)\right) j .-$ Gate AND. Let $j$ $=\operatorname{depth}(\mathrm{w})$. This algorithm decides arbitrary $\mathrm{aw}, \mathrm{b}_{\mathrm{w}} \in \mathrm{Z}_{\mathrm{p}}$. The splits are: $\mathrm{C}_{\mathrm{w}} ; 1=\mathrm{g}^{\mathrm{aw}}, \mathrm{C}_{\mathrm{w}} ; 2=\mathrm{g}{ }^{\mathrm{a}}\left(\mathrm{r}_{\mathrm{w}}-\mathrm{a}_{\mathrm{w}} \mathrm{r}_{\mathrm{A}}(\mathrm{w})\right.$ $\left.-b_{w} r_{B}(w)\right) j$. For the OR and AND gates in circuit $f$, the sharing techniques are as the same as in $f$. When cancellation gates emerge in the input level, setting $\mathrm{f}_{\mathrm{w}}(\mathrm{x})$ $=\mathrm{x}_{\mathrm{w}}$, the splits of the equivalent input wire $\mathrm{w}$ will be: $\mathrm{C}_{\mathrm{w}}$, $=y^{\mathrm{r}}{ }_{\mathrm{w}} \mathrm{h}^{-\mathrm{zw}}{ }_{\mathrm{n}+\mathrm{w}}, \mathrm{C}_{\mathrm{w}, 2}=\mathrm{g}^{\mathrm{zw}}$. Then we could consume the circuit $f$ to split the encryption proponent s2. The complete ciphertext $\mathrm{CT}$ includes $\mathrm{C}_{\mathrm{M}}, \mathrm{C}^{\prime}{ }_{\mathrm{M}}, \mathrm{C}_{\mathrm{R}}, \mathrm{C}^{\prime}{ }_{\mathrm{R}}, \sigma$, the ciphertext of $f$ and $\left(\mathrm{C}^{\prime}{ }_{\mathrm{M}}, \mathrm{C}^{\prime} \mathrm{R}\right.$, the ciphertext of $\mathrm{f}$ and $\mathrm{f}$ are measured as the KEM part indicated by $\left(\mathrm{CK}_{\mathrm{M}}, \mathrm{CK}_{\mathrm{R}}\right)$. $\left(\mathrm{C}_{\mathrm{M}}, \mathrm{C}_{\mathrm{R}}, \sigma\right)$ is measured as the AE part). In summary, the total ciphertext for our VD-CPABE system is the tuple CT $=\left(\mathrm{CK}_{\mathrm{M}}, \mathrm{CK}_{\mathrm{R}}, \mathrm{C}_{\mathrm{M}}, \mathrm{C}_{\mathrm{R}}, \sigma_{\mathrm{M}}, \sigma_{\mathrm{R}}\right)$

- KeyGen (MK, $x \in\{0,1\} n)$ The influence produces the private key for the consumer. Then the consumer flings his alteration key to the cloud server. This algorithm obtains as input the master secret key and a explanation of the characteristic $\mathrm{x} \in\{0,1\} \mathrm{n}$. It firstly decides an arbitrary $\mathrm{t} \in \mathrm{Zp}$. Then it makes the private key and conversion key .Note that, for the data owner IDo, the influence produces his private key with the self quality ID0.

- Transform (TK, CT). The conversion algorithm is implemented by the cloud server. It obtains as input the conversion key TK and the innovative ciphertext CT. The algorithm incompletely decrypts the ciphertext as follows. Taking TK with $x$ as input, we estimate the circuit from the substructure positive. If $\mathrm{f}(\mathrm{x})=1$ we will be clever to incompletely decrypt the ciphertext for $\mathrm{M}$ and if $\mathrm{f}(\mathrm{x})=0$ we will be clever to incompletely decrypt the ciphertext for $\mathrm{R}$. regard as the wire $\mathrm{w}$ at deepness $\mathrm{j}$, if $\mathrm{f}_{\mathrm{w}}(\mathrm{x})=1$ then the algorithm calculates $E_{w}=\left(g_{j+1}\right)^{\text {arwt }}$ and if $f_{w}(x)=0$ the algorithm measure naught. The estimate depends on if $\mathrm{w}$ is an Input wire, an OR gate, or an AND gate. These are all the fractional decryption algorithms.

\section{SECURITY ANALYSIS}

In our proposed fusion VD-CPABE system, the AE fraction is executed by a one-time symmetric-key encryption and the encrypt-then-Mac model. $(\mathrm{C}, \sigma)$ is measured as the IND-CCA safe AE fraction. The subsequent theorem shows that the KEM part is INDCPA protected. Presume there survives a PPT invader $\mathrm{A}$ in our KEM technique for a circuit of depth and inputs of length $\mathrm{n}$ in the discriminating selected plaintext safety game; we can create a PPT algorithm that resolves the multi linear supposition with non-negligible benefits. Denotation depends this instantly illustrates that the challenger A1 with non-trivial benefits in the KEM refuge game will have an indistinguishable leads in flouting the k-MDDH statement. In below we demonstrate the graph for analyzing the security of the system. 


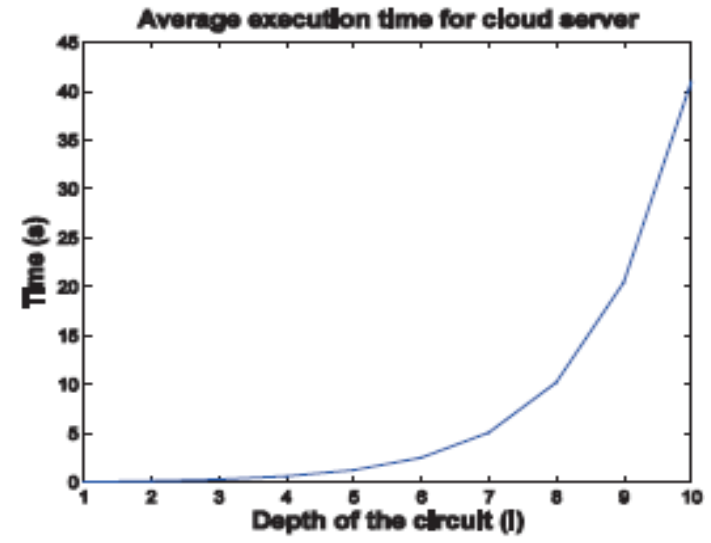

Figure. 3. Performance of our hybrid VD-CPABE Scheme

In $\mathrm{x}$ axis we denote circuit depth and in $\mathrm{y}$ axis time is denoted. By these two entities we can find the average execution time for cloud server. This graph's slope denotes the performance and security analysis of the circuit cipher text model.

\section{IMPLEMENTATION}

In this segment, we replicate the cryptographic procedures. Without allowing for the calculation of two essentials above the numeral hash function and an exclusive- OR operations, we indicate the value of a multi linear combination by P. $\lambda$ indicates the defense constraints. $\beta$ indicates the set essentials size in small pieces. With dissimilar constraints, the standard operation instance of operations. When we activate the encryption and fractional decryption algorithms, the input wire and the AND gate require to jumble twice and the OR gate desires to jumble triple. The algorithm for making MAC requires one jumbling action and other further operations in excess of the integer, and the algorithm for validating MAC requires jumbling triple. Based on the exceeding constraint settings, the majority administration instance to terminate our encryption and decryption algorithm is demonstrated. The bandwidth of the broadcasted ciphertext for the data owner develops with enlarges of the depths of despair of circuit. For the consumer, the bandwidth of the broadcasted ciphertext is calculated. Apparently, for the data owner and the cloud server, the calculation time increases proponent ally with enlarge of the intensity of circuit. When depth, these calculations are billed respectively. Thus our system facilitates to offer proficient technique to split and defend the secret information between users with inadequate power and data owners with enormous amount of data in the cloud.

\section{CONCLUTION}

To the best of our knowledge, we initially here a circuit ciphertext-policy attribute-based hybrid encryption with provable allocation method. Universal circuits are helpful to articulate the strongest form of entrée manage strategy. Collective provable calculation and encrypt-then-Mac system with our ciphertext policy attribute-based hybrid encryption, we could hand over the provable fractional decryption paradigm to the cloud server. Within calculation, the proposed system is established to be safe based on k-multi linear
Decisional Diffie-Hellman supposition. On the other hand, we execute our scheme above the integers. The expenses of the calculation and announcement expenditure show that the method is sensible in the cloud computing. Thus, we could be relevant it to create confident the data privacy, the fine-grained entrée manages and the demonstrable allocation in cloud.

\section{REFERENCES}

[1] B. Waters," Ciphertext-Policy Attribute-Based Encryption: An Expressive, Enficient, and Provably Secure Realization,” in Proc. PKC, pp.53-70, SpringerVerlag Berlin, Heidelberg, 2011.

[2] Bryan Parno, Mariana Raykova, and Vinod Vaikuntanathan. How to delegate and verify in public: Verifiable computation from attribute-based encryption. In TCC, pages 422-439, 2012.

[3] S. Yamada, N. Attrapadung and B. Santoso," Verifiable Predicate Encryption and Applications to CCA Security and Anonymous Predicate Authentication," in Proc. PKC, pp.243-261, Springer-Verlag Berlin, Heidelberg, 2012.

[4] T. Granlund and the GMP development team, "GNU MP: The GNU Multiple Precision Arithmetic Library, 5.1.1," 2013, http://gmplib.org/.

[5] J. Coron, T. Lepoint and M. Tibouchi," Practical Multilinear Maps over the Integer," in Proc. CRYPTO, pp.476-493, Springer-Verlag Berlin, Heidelberg, 2013.

[6] V. Goyal, O. Pandey, A. Sahai and B. Waters," Attributebased Encryption for Fine-grained access control of encrypted data," in Proc. CCS, pp.89-98, ACM New York, NY, USA, 2006.

[7] M. Abe, R. Genaro and K. Kurosawa," Tag-KEM/DEM: A New Framework for Hybrid Encryption," in Proc. CRYPTO, pp.97-130, Springer-Verlag New York, NJ, USA, 2008.

[8] S. Garg, C. Gentry and Shai Halevi," Candidate Mulitilinear Maps from Ideal Lattices and Applications," in Proc. EUROCRYPT, pp.1-17, Springer-Verlag Berlin, Heidelberg, 2013.

[9] Melissa Chase and Sherman S. M. Chow. Improving privacy and security in multiauthority attribute-based encryption. In ACM Conference on Computer and Communications Security, pages 121-130, 2009.

[10] Jonathan Katz, Amit Sahai, and Brent Waters. Predicate encryption supporting disjunctions, polynomial equations, and inner products. In EUROCRYPT, pages 146-162, 2008 .

[11] M. Green, S. Hohenberger and B. Waters," Outsourcing the Decryption of ABE Cipher texts," in Proc. USENIX Security Symp., San Francisco, CA, USA, 2011.

[12] Tatsuaki Okamoto and Katsuyuki Takashima. Adaptively attribute-hiding (hierarchical) inner product encryption. In EUROCRYPT, pages 591-608, 2012. 